\title{
Gestión de las actividades turísticas en los municipios de Licey Almedio y Tamboril en su desarrollo sostenible
}

\author{
José Alberto Acosta Guzmán \\ UTESA - Rep. Dominicana \\ josacoguz@hotmail.com
}

Recibido: 23-01-2015

Aceptado: 23-02-2015

Keywords: Tourism management, communication, sustainable, towns, rural

\section{ABSTRACT}

The growth of rural tourism in the Dominican Republic eminently tourist, whose specialization in tourism called "sun and beach" is being heretofore virtually all, this poses significant challenges in management, particularly in the area of marketing. The characteristics of this type of tourism and the need to preserve and conserve the environment in which it develops, determine the actions to be carried out in this area and require adequate planning that takes into account not only the benefits of this tourism, but also the expenses generated. In this research the factors to consider when implementing tourism activities in the rural environment (Licey -Tamboril) and the need to assess the impacts, both positive and negative, this would generate in the area are analyzed. Also, the communication factor is involved, to understand that their role is basically the sustainable growth of rural tourism. In this research very important issues for Licey munícipes Almedio and Tambourine, which are adjacent, primarily by taxis called DE LA PENA, have in common, a natural resource of great importance and this represents the tree will be treated the Ceiba, which has over a thousand years, representing the oldest West Indian natural resource.

\begin{abstract}
Palabras claves: Gestión turística; comunicación; sostenible; municipios; Rural.
\end{abstract}

\section{RESUMEN}

El crecimiento del turismo rural en la República Dominicana eminentemente turístico, cuya especialización en el denominado turismo de "sol y playa" viene siendo hasta este momento prácticamente en lo absoluto, esto plantea importantes desafios en su gestión, particularmente en el área de marketing. Las características de esta tipología turística y la necesidad de preservar y conservar el medio ambiente en el que se desarrolla, condicionan las acciones a llevar a cabo en este ámbito y exigen una adecuada planeación que tenga en cuenta no sólo los beneficios derivados de esta actividad turística, sino también los gastos que genera. En este trabajo de investigación se analizan los factores a considerar a la hora de implementar acciones turísticas en el medio ambiente rural (Licey -Tamboril) y la necesidad de evaluar los impactos, tanto positivos como negativos, que ello generaría en la zona. Asimismo, se tratara el factor comunicación, por entender que su rol es básicamente el crecimiento sostenible del turismo rural. En esta investigación se trataran cuestiones muy importantes para los munícipes de Licey Almedio y Tamboril, los cuales, son colindantes, fundamentalmente por la carretea denominada DE LA PEÑA, tienen en común, un recurso natural de mucha importancia y esto lo representa el árbol de 
la Ceiba, el cual tiene más de mil años, representando el recurso natural más antiguo de las Antillas.

\section{INTRODUCCION}

En la literatura actual acerca del turismo rural, como fenómeno económico, social y cultural, por lo general, es frecuente encontrar continuas referencias al impacto que éste tiene sobre las zonas geográficas en las que se desarrolla. Así, son muchos los expertos en la materia, que han expresado los grandes peligros que puede, y de hecho está ya acarreando en algunos lugares, el aumento masivo de visitantes.

La necesidad de realizar un cambio en el denominado turismo de masas, básicamente el de sol y playa, tratando de lograr la armonía con el medio ambiente y la población, implica asumir responsabilidades sociales, morales y ecológicas, que pongan freno al crecimiento desmedido e incontrolado de la actividad turistica en determinadas zonas rurales. Esta situación, que sin duda es del todo cierto, que todas las partes implicadas están tomando conciencia, supone una preocupación, aún si cabe más importante, en el caso del turismo rural. Esta nueva demanda turística el disfrute del medio ambiente es siempre la motivación esencial y toda la actividad turistica se realiza en contacto con dicho medio. Se impone, por tanto, la necesidad de preservarlo de toda acción perjudicial para su conservación en perfectas condiciones de disfrute en el tiempo.

Por lo antes expresado, esta investigación busca, en primer lugar, determinar los factores a considerar a la hora de planificar cualquier plan de turismo rural, con el objetivo de lograr un desarrollo sostenible de esta actividad, en este caso los gestores que intervienen: el Ministerio de Medio Ambiente y el Ministerio de Turismo, fundamentalmente el departamento que conjuga estos dos ministerios el Ecoturismo.

\section{LA GESTIÓN DE LA ACTIVIDAD TURISTICA EN LA ZONA RURAL}

En el momento de gestionar cualquier actuación en las zonas rurales (Licey -Tamboril), hay que partir de la necesidad que permitan el establecimiento de un punto de equilibrio entre lo que el visitante demanda y las condiciones que posee y puede ofertar una determinada zona rural, para ser capaz de soportar dicha demanda. Se trata de mensurar la rentabilidad social de los planes turísticos en la zona rural (Casanueva, García del Junco y Caro, 2000).

Se debe tener en cuenta las investigaciones de los experto en esta área los cuales propones la primera explicación a esta temática, Cals (1996), en contraposición con la rentabilidad de los sectores privados. Así, la rentabilidad social considera además de los ingresos y gastos privados. La rentabilidad social se calcula, por tanto, como la satisfacer sus propias necesidades" Se trata, por tanto, de satisfacer los deseos y necesidades de la demanda, pero asegurando el mantenimiento a lo largo plazo la oferta. A partir de este concepto de desarrollo sostenible, aparece la noción de "turismo sostenible" que es definido por Reguero (1994) como el "turismo cuyas acciones están integradas en el entorno junto con la población local, y buscan una explotación en el largo plazo evitando un modelo de máxima rentabilidad en el tiempo y en el espacio propio de la especulación, que tan común ha sido en el turismo tradicional". 
Esto indica y reafirma, que antes de lazar cualquier plan turístico, se requiere de una exhaustiva evaluación. Esta debe efectuarse, tal y como se ha señalado en los párrafos anteriores, teniendo en consideración los resultados económicos y medioambientales, así como culturales y sociales. Sin embargo, es a la hora de de la praxis estos conocimientos teóricos, cuando surgen algunos inconvenientes relacionados, básicamente, a la dificultad para cuantificar tanto los impactos positivos como los negativos, que genera la actividad turística en el medio rural. Esta cuantificación es un paso básico en la planeación de ese medio turístico rural, ya que en función del resultado el plan seguirá adelante o, por el contrario, será rechazado.

Entre los métodos a los que hacemos referencia destacamos el que ha propuesto Opaschowski (1990), que establece un "balance ecológico del ocio y del turismo". Este método se fundamenta en la idea de que la industria turistica y del ocio ha de tener en consideración las consecuencias que tienen sus actividades económicas en el medio ambiente, como gastos para el futuro. Así, Opaschowiski establece que "un tipo de proporción gasto-beneficio ha de determinar el consumo de recursos de cualquier plan de recreo, cerciorándose de los resultados en los cambios del medio ambiente, para así calcular los gastos resultantes de los daños ecológicos en el aire, agua y suelo. De este modo, puede averiguarse qué tipo de inversiones adicionales en recreo e industria turística pueden y deben llevarse a cabo para garantizar el desahogo en el medio ambiente".

Se debe comprender, que es en este punto, donde inicia la verdadera labor del empresario rural, diseñando y comercializando una oferta no sólo adecuada para los visitantes seleccionados y para el logro de sus propios objetivos sino también, acorde con las limitaciones y parámetros previamente establecidos por la actuación del estado dominicano. Casillas, Moreno y Oviedo (1995), indican en este mismo orden, la necesaria planeación de esta clasificación de turismo que fundamentalmente se concretaria en dos grandes corrientes de actuación: la creación de las infraestructuras necesarias para la minimización de los impactos físicos negativos del turismo, así como la potenciación de los impactos positivos. En otro orden de ideas, la transmisión de valores a las partes, directa o indirectamente implicadas en este desarrollo turístico, es decir, la educación de dichas partes en su actuación sobre el entorno.

En cuanto a las infraestructuras existentes, se entienden como el conjunto de elementos humanos y materiales que dentro del turismo rural están destinadas a:

1.- Reducir el impacto negativo de los turistas en la zona: éste se puede alcanzar procediendo sobre dos elementos básicos: la naturaleza y el visitante. En el primer caso, habría que dotar al área turística con aquellos elementos materiales que impidan la acción del hombre sobre la naturaleza, orienten su actuación, restablezcan las condiciones previas a la visita y prevengan posibles accidentes derivados de las visitas.

2.- Proporcionar beneficios variados de ocio adecuados al objetivo de lo planificado. Así, esta oferta turística debe ser: diversificada, flexible y proporcionar recursos económicos existentes en la zona rural. Con relación a la educación, ésta consistiría en crear mediante actuaciones educativas una conciencia respetuosa en el entorno rural. Esta actividad se dirigiria tanto a los visitantes como a las comunidades locales. Los primeros actúan casi siempre exclusivamente como receptores, los segundos, en cambio, pueden ser receptores pero también emisores pasivos, a través de la distribución de material elaborado por el estado dominicano. Esta función educativa puede desarrollarse de dos formas: a través de una comunicación individualizada entre los guías de la zona ampliamente formados y los turistas o bien utilizando formas de comunicación impersonal. 
Además, se puede destacar, que los medios de comunicación masivos, dirigidos a la población en general y que, entre otras cosas, pueden pretender proporcionar una formación genérica de respeto al entorno o dar a conocer las distintas ofertas existentes. Por otro lado, estarian los medios de comunicación dirigidos a los turistas y cuyo mensaje iría encaminado a educarles en su comportamiento durante la visita a la zona rural. Esta información puede suministrarse en los puntos de acceso al área a través de folletos, carteles o bien en su interior, a través de zonas de descanso.

Se puede, en consecuencia observar, la relevancia que las acciones de comunicación tienen en este proceso. Estas actuaciones deberán ser tanto para el sector público como privado, ya que si la labor de comunicación que realizan las distintas gestiones es destacable, también tiene que serlo la comunicación que desarrollen las empresas privadas. Estas, buscarán informar al visitante de cada oferta en específico y "formarle", en cierta medida, para que su actuación en el medio rural (Licey -Tamboril) sea adecuada y contribuya así a mantener la zona en las condiciones deseadas; circunstancia ésta que entendemos puede generar importantes ventajas competitivas a las empresas que lo logren y garantizar su supervivencia a largo plazo.

En este sentido se puede expresar, que antes de dar inicios a cualquier actividad turística en el medio rural, es necesaria una utilización combinada de los distintos análisis existentes e incluso, si es necesario, desarrollar una metodología específica para cada actividad a realizar. Tomando siempre en consideración, que cada zona rural posee sus propias características sociales, económicas, culturales, demográficas y medioambientales, siendo necesario establecer medidas específicas para cada una de esas zonas. Para ello, un primer paso debe ser la determinación de aquellos indicadores de turismo sostenible que se consideren más apropiados para cada plan en cuestión y proceder a su formulación y evaluación.

Teniendo estas informaciones, por consiguiente, se puede establecer un listado de todos aquellos factores susceptibles de verse afectados, tanto positiva como negativamente, por el plan a desarrollar. También, y dado que la actuación del turismo tendrá diferentes resultados sobre cada uno de ellos, siendo algunos considerados más importantes que otros, se podría asignar una ponderación a cada factor escogido para su análisis.

En definitiva, se puede afirmar, que toda formulación y evaluación del plan, debe realizarse estableciendo previamente las prioridades de cada zona rural (Licey -Tamboril) bajo estudio. Sólo así se podrá conseguir un turismo rural que fundamente su desarrollo en la calidad y no en la cantidad, que sea rentable y además, social y medioambientalmente aceptable. En el ámbito de las empresas ofertantes de servicios turísticos rurales, la función de marketing relacional debe llevarse a cabo teniendo en consideración las limitaciones que se han señalado. Dado todo lo expuesto con anterioridad, se puede considerar que un factor fundamental sobre la que se debe incidir desde el área de marketing es la comunicación.

\section{LA COMUNICACIÓN COMO FACTOR DE CRECIMIENTO SOSTENIBLE DEL TURISMO RURAL}

El turístico representa un sector fundamentalmente de servicios, la importancia de una buena comunicación. Así, el visitante se encuentra con una serie de circunstancias, derivadas en parte de la intangibilidad del servicio, que hacen que el riesgo asociado a su decisión sea mayor que en el caso de los productos tangibles. En estas condiciones, la comunicación que de los servicios turísticos se realice contribuirá a "tangibilizar" en parte dichos servicios, aportando 
información al visitante, que facilitarle las cosas en el proceso de elección, del destino turístico rural(Licey -Tamboril). Esto implica, el establecimientos de mecanismos de comunicación, que faciliten el proceso de escogencia del destino turístico rural, permitiendo a los visitantes, claridad el en la utilización de esas informaciones.

La comunicación en el sector turístico supone, por tanto, un importante instrumento de marketing a disposición de los destinos y empresas turísticas, para crear preferencia hacia sus productos o servicios, frente a otros competidores. Con lo antes expuesto, se puede afirmar, que uno de los factores más relevantes de todo el proceso de comunicación turística rural es el mensaje que se transmite y éste, en algunos casos, adolece de ciertas deficiencias en su contenido que hacen que el comportamiento de los visitantes no se produzca en el sentido deseado. Rodriguez-Del Bosque (1996), en referencia a las instituciones turísticas, indica que la comunicación dispone de dos mecanismos de actuación sobre el comportamiento del consumidor: En primer lugar, un componente informativo que proporciona al visitante un mayor conocimiento a través de la información suministrada. En segundo lugar, un componente persuasivo para convencer a los visitantes. Este proceso de persuasión se puede conseguir a través de tres posibles vías:

$>\quad$ La racionalidad, en la que se enfatiza en aspectos tangibles y demostrables del servicio turístico, en este caso, la visita a la casa de Trina de Moya y Horacio Vásquez o la visita a la Ceiba, el árbol con más de mil años.

$>\quad$ La emocional, en la que se trata de persuadir a través de mensajes que lleguen a las fibras más sensibles del visitante.

La inconsciente, en la que los mensajes tratan de alcanzar el subconsciente del turista y empujarle a la acción.

En un sentido amplio, en todo tipo de comunicación y, por tanto, también en la perteneciente al sector turístico dominicano, el componente informativo y el persuasivo se unen buscando la respuesta deseada por parte del visitante. En la situación del turismo rural, se puede entender que la comunicación que se viene desarrollando en la actualidad, ante todo es informativa. Así, el estudio de los medios más empleados en este mercado: folletos, guías y páginas en internet, han demostrado que dicha comunicación se fundamenta en la transmisión de mensajes orientados a que el visitante conozca aspectos funcionales de las ofertas de turismo rural, tales como precios, ubicación y características del alojamiento.

Ahora se debe aclara, como lo ha explicado Traverso (1996) de que el visitante, a través de la comunicación que recibe, comprenda mejor lo que se le ofrece y sepa actuar según los parámetros deseados. En último término esta comunicación formativa trataria de lograr la involucración del visitante en la consecución de planes establecidos, a través de un proceso de adaptación, comprensión y respeto de la zona rural que visita, así como de sus residentes.

Se puede entender, que la comunicación tiene la suficiente entidad y relevancia para el crecimiento del turismo rural, como para ser considerada a la hora de planificar cualquier acción de comunicación. En otro orden de ideas, se puede considerar, que una comunicación como la descrita va más allá de la mera información al visitante. De esta manera, no sólo se conseguirá una respuesta positiva por parte del visitante, sino que además se logrará que responda de acuerdo con los parámetros establecidos con anterioridad. Formación, información y persuasión deben, por tanto, actuar de forma conjunta para provocar dicha respuesta. Es evidente que esta labor de formación no se consigue a corto plazo, sino que exige tiempo y recursos económicos para abordar todo el proceso y alcanzar este ambicioso objetivo (largo plazo). La importancia del visitante junto con la preocupación por el desarrollo sostenible de la 
zona rural (Licey -Tamboril), harán de este modelo de comunicación un elemento clave para el éxito del turismo rural.

A continuación se produce la explicación de la comunicación, que debe producirse de forma original. A través de la explicación el visitante que llegue a ciudadela de Tamboril o Licey descubrirá la relevancia del mensaje y la forma en la que puede beneficiarse de la información interpretada. Tendrá que hacer un esfuerzo, igualmente, en transmitir un mensaje coherente de tal manera que todos los elementos que intervienen en el programa de comunicación: colores, vestuario, música, estén coordinados para lograr una comunicación global. Finalmente, hay que ofrecer una visión de conjunto centrando la interpretación en un punto o tema principal. Se trata, en definitiva, de establecer aquel o aquellos aspectos del lugar visitado que se desea sean aprendidos o recordados por el visitante. La articulación del modelo de comunicación interpretativa o comunicación formativa, puede estructurarse según Veverka (1994) en cinco fases:

1.- Primera fase se efectuaría un inventario de los recursos humanos, tecnológicos, culturales, patrimoniales y medio-ambientales que posee la zona rural para desarrollar asi el tema principal a transmitir al visitante.

2.- Segunda etapa, una vez que sepamos el mensaje que queremos transmitir a los visitantes, se desarrollarán los objetivos interpretativos específicos que pretendemos lograr. Estos objetivos son la base de las acciones siguientes y sirven para la evaluación posterior.

Un objetivo interpretativo debe expresar lo que se espera que el visitante aprenda, sienta o haga y pueden distinguirse básicamente tres tipos (Veverka, 1994):

\begin{tabular}{|l|l|}
\hline Elementos & Descripción \\
\hline Objetivos de aprendizaje & $\begin{array}{l}\text { Definen aquellos elementos o actuaciones } \\
\text { que se desea que el visitante aprenda o } \\
\text { identifique }\end{array}$ \\
\hline Objetivos de comportamiento: & $\begin{array}{l}\text { Buscan obtener una actuación o } \\
\text { comportamiento concreto por parte del } \\
\text { turista. }\end{array}$ \\
\hline Objetivos emotivos & $\begin{array}{l}\text { Los objetivos de comportamiento no se } \\
\text { podrán alcanzar normalmente, si no se } \\
\text { consigue implicar emocionalmente al } \\
\text { turista. }\end{array}$ \\
\hline
\end{tabular}

Fuente: Elaboración propia a partir de Veverka.

3.- Se debe analizar el mercado de forma paralela con el objetivo de conocer el perfil de los distintos tipos de visitantes a las zonas de Licey y Tamboril y poder desarrollar así los programas y servicios que mejor se adapten a las necesidades de cada segmento detectado.

4.- Desarrollar los aspectos operativos de la planificación, decidiendo la programación y contenidos, es decir, codificación de la comunicación, los medios más adecuados y los costes que conlleva.

5.- Establece formas de evaluación que permitan establecer si se están alcanzando los objetivos fijados. 


\section{CONCLUSIONES}

Finalizada esta investigación, se puede afirmar, que al momento de planificar cualquier actividad turística en el medio rural es importante y necesario proceder previamente al análisis de las repercusiones económicas, sociales, culturales y medioambientales, que tal actuación supondrá en el entorno rural. Si nos centramos, en las repercusiones medioambientales, las acciones de comunicación que lleven a cabo tanto las empresas privadas como las instituciones estatales, pueden hacer que la actuación de los visitantes contribuya, en gran medida, al crecimiento sostenible del turismo rural (Licey - Tamboril). Esto se puede lograr mediante la comunicación formativa, que se realiza antes y durante su visita a la zona rural, se informa al turista de lo que va a recibir, también se hace referencia a cómo tiene que ser su actuación en el entorno rural visitado y por qué. El visitante conoce así, antes de iniciar su viaje, qué comportamiento se espera de él, no sólo en cuanto a lo que no debe hacer sino también, y tal vez más importante, en cuanto a lo que puede o debe hacer para contribuir a preservar por mucho tiempo la actual situación de la zona e incluso, si es posible, mejorarla.

La institución turística rural tiene que lograr con su efectiva comunicación que el visitante se identifique con el entorno rural que visita, que no se sienta alejado de sus residentes y sus tradiciones y entienda qué papel debe desempeñar además, claro está, de disfrutar de su tiempo de ocio.

Thibal (1983), ha expresado, que se debe logran en los visitantes, no se sientan como una mera fuente de ingresos para el área rural y que los residentes locales no se sientan como un objeto a fotografiar. Se debe entender, que lograr esta meta no es tarea fácil, pero también se debe considerar que si se enfoca la comunicación hacia el visitante de tal manera que no se le transmitan estas ideas, se puede correr el riesgo de limitar la vida del turismo rural al degradarse los factores claves de éxito, que constituyen la base de su disfrute. Pero la labor de comunicación no finaliza cuando el turista ha adquirido su viaje sino que tiene que continuar en el lugar de su disfrute. Allí, serán fundamentalmente los prestadores de servicios y los residentes locales los que, evidentemente a través de otros métodos, logren seguir informando, formando e integrando al turista en el medio rural escogido (Licey -Tamboril).

Finalmente, se puede afirmar, que en la mayoria de las situaciones, esta labor de formación tendrá que llevarse a cabo previamente con los propios oferentes y residentes rurales para que entiendan cómo deben actuar con los visitantes y los beneficios que obtendrán de ello. Se trata, en definitiva, de una labor de comunicación interna sin la cual dificilmente se podría desarrollar el modelo de comunicación propuesto. En consecuencia, un completo sistema de comunicación, en el que cada factor desempeña un papel decisivo en el engranaje del turismo rural y contribuye a formar una buena imagen en la mente del turista.

\section{BIBLIOGRAFIA}

Cals Güel, J. (1996): "La rentabilidad social de los proyectos turísticos" en Valdés Peláez, L. y Ruiz Vega, A. V. (coord.): Turismo y promoción de destinos turísticos. Universidad de Oviedo. Servicio de Publicaciones. Gijón, pp. 225-240

Casanuevas, C.; García del Junco, J y Caro, F. J. (2000): Organización y gestión de empresas turísticas. Pirámide. Madrid.

Casillas, J. C.; Moreno, A. M. y Oviedo, M. A. (1995): "El turismo alternativo como un sistema integrado: consideraciones sobre el caso andaluz". Estudios turísticos, n ${ }^{\circ} 125$, pp. 53-74. 
Comisión Mundial sobre medio Ambiente y Desarrollo. W.C.E.D. (1987): Our Commom Future. Oxford University Press. Melbourne.

Opaschowski, H. W. (1990): “¿Turismo de masas o turismo a medida?. Límites económicos, ecológicos y psicológicos". Papers de turisme, $\mathrm{n}^{\circ} 4$, pp. 68-80.

Reguero Oxinalde, M. del (1994): Ecoturismo. Nuevas formas de turismo en el espacio rural. Bosch Turismo. Barcelona.

Rodríguez - Del Bosque, I.A. (1996): "La comunicación de los servicios turísticos" en Valdés Peláez, L. y Ruiz Vega, A. V. (coord.): Turismo y promoción de destinos turísticos. Universidad de Oviedo. Servicio de Publicaciones. Gijón, pp. 175-202.

Thibal, S. (1983): "Tourisme en espace rural et tourisme de pays en developpement: un même problème de comunication sociale". Revue de Tourisme, $\mathrm{n}^{\circ} 4$, pp. 18-21.

Traverso Cortés, J. (1996): "Comunicación interpretativa: variable clave en el marketing-mix de las empresas de turismo rural”. Estudios Turísticos, n 130, pp. 37-50.

Veverka, J.A. (1994b): "Desarrollo del plan interpretativo: visión de conjunto de la estrategia básica de planificación”, en Crosby, A. (dir.): Interpretación ambiental y turismo rural. Centro Europeo de Formación Ambiental y Turística. Madrid, pp. 27-36.

\section{HOW TO CITE THIS ARTICLE IN BIBLIOGRAPHIE}

Acosta Guzmán, J. A. (2015): "Gestión de las actividades turísticas en los municipios de Licey Almedio y Tamboril en su desarrollo sostenible" Rotur. Revista de Ocio y Turismo, 8: 1-8, ISSN-e: 2695-6357, DOI: https://doi.org/10.17979/rotur.2015.8.1.1292 